\title{
A Novel Type of Macrothrombocytopenia Associated with a Defect in $\alpha 2,3$-Sialylation
}

\author{
Claire Jones, ${ }^{\star \dagger}$ Jonas Denecke, ${ }^{\ddagger}$ Ronald Sträter, ${ }^{\ddagger}$ \\ Torsten Stölting, ${ }^{\ddagger}$ Yvonne Schunicht, ${ }^{*}$ \\ Dagmar Zeuschner, ${ }^{*}$ Judith Klumperman, ${ }^{\S}$ \\ Dirk J. Lefeber, " Oliver Spelten, ${ }^{* \|}$ \\ Alexander Zarbock, ${ }^{*, * *}$ Sørge Kelm, ${ }^{\dagger \dagger}$ \\ Karen Strenge, ${ }^{\text {†† }}$ Stuart M. Haslam, ${ }^{\neq+}$ \\ Kerstin Lühn, ${ }^{\S \S}$ Dorothea Stahl, ${ }^{, \uparrow 1|||| ~ L u c a ~ G e n t i l e, ~}{ }^{*}$ \\ Thomas Schreiter, ${ }^{* \star *}$ Philip Hilgard, ${ }^{+\dagger \dagger}$ \\ Annette G. Beck-Sickinger, \\ Thorsten Marquardt, ${ }^{\ddagger}$ and Martin K. Wild ${ }^{\star}$ \\ From the Max Planck Institute for Molecular Biomedicine," \\ Münster, Germany; the Universitätsklinikum Münster, ${ }^{\ddagger}$ Klinik für \\ Kinder- und Jugendmedizin, Münster, Germany; the Oxford \\ Vaccine Group, Department of Paediatrics, ${ }^{\dagger}$ and the Weatherall \\ Institute of Molecular Medicine, MRC Human Immunology \\ Unit, ${ }^{\$ S}$ University of Oxford, Oxford, United Kingdom; the \\ Department of Cell Biology, ${ }^{\circledR}$ University Medical Center, Utrecht, \\ the Netherlands; the Department of Neurology, Department of \\ Laboratory Medicine, ${ }^{\pi}$ Radboud University Nijmegen Medical \\ Centre, Nijmegen, the Netherlands; the Department of \\ Anesthesiology and Postoperative Intensive Care Medicine," \\ University of Cologne, Cologne, Germany; the Department of \\ Anesthesiology and Critical Care Medicine, "** University of \\ Münster, Münster, Germany; the Centre for Biomolecular \\ Interactions Bremen, ${ }^{+\dagger}$ University of Bremen, Bremen, Germany; \\ the Division of Molecular Biosciences, \\ London, United Kingdom; the University Clinic for Blood Group \\ Serology and Transfusion Medicine, ${ }^{\text {वाा }}$ Paracelsus Medical \\ University, Salzburg, Austria; Haema AG, IIII Leipzig, Germany; the \\ Department for Gastroenterology and Hepatology,*** University \\ Hospital Essen, Essen, Germany; Evangelisches Krankenhaus \\ Mülheim, ${ }^{+t \dagger}$ Mülheim, Germany; and the Institute of \\ Biochemistry, ${ }^{\ddagger \neq}$ Leipzig University, Leipzig, Germany
}

We describe a novel type of human thrombocytopenia characterized by the appearance of giant platelets and variable neutropenia. Searching for the molecular defect, we found that neutrophils had strongly reduced sialyl-Lewis $X$ and increased Lewis $X$ surface expression, pointing to a deficiency in sialylation. We show that the glycosylation defect is restricted to $\alpha 2,3$-sialylation and can be detected in platelets, neutrophils, and monocytes. Platelets exhibited a dis- torted structure of the open canalicular system, indicating defective platelet generation. Importantly, patient platelets, but not normal platelets, bound to the asialoglycoprotein receptor (ASGP-R), a liver cellsurface protein that removes desialylated thrombocytes from the circulation in mice. Taken together, this is the first type of human thrombocytopenia in which a specific defect of $\alpha 2,3$-sialylation and an induction of platelet binding to the liver ASGP-R could be detected. (Am J Pathol 2011, 179:1969-1977; DOI: 10.1016/j.ajpath.2011.06.012)

Several types of hereditary macrothrombocytopenia have been described, including Bernard-Soulier syndrome and the May-Hegglin anomaly, which are caused by mutations in the genes coding for platelet glycoproteins (GP) Ib/IX and nonmuscle myosin heavy chain, respectively. ${ }^{1,2}$ Macrothrombocytopenia can also be caused by a defect in glycosylation. ${ }^{3}$ This disease, termed congenital disorder of glycosylation-IIf (CDG-IIf), was detected in a child whose neutrophils lacked expression of the sialic acid-containing tetrasaccharide sialyl-Lewis $X\left(s \mathrm{Le}^{\mathrm{x}}\right)$ and showed increased expression of its nonsialylated form Lewis X $\left(\mathrm{Le}^{\times}\right)$. Notably, abnormal demarcation membranes in megakaryocytes strongly pointed to a defect in the generation of thrombocytes. $^{3}$ Mutations in the gene encoding the Golgi transporter for cytidine monophosphate (CMP)-sialic acid were described as the cause for this disease. ${ }^{4}$

Generally, sialylation appears to strongly affect the number of circulating platelets. Indeed, desialylation of

Supported by the Max Planck Society (M.K.W. and C.J.), by BMBF and IMF (J.D., T.St., and T.M.), by DFG (AZ428/3-1 to A.Z.), and by Volkswagenstiftung, HFSP and BMBF (S.K. and K.S.)

Accepted for publication June 3, 2011.

T.M. and M.K.W. contributed equally as senior authors of this work.

Supplemental material for this article can be found at http://ajp. amjpathol.org or at doi: 10.1016/j.ajpath.2011.06.012.

Address reprint requests to Martin K. Wild, Ph.D., Max Planck Institute for Molecular Biomedicine, Röntgenstr. 20, 48149 Münster, Germany or Thorsten Marquardt, M.D., Universitätsklinikum Münster, Klinik für Kinder- und Jugendmedizin, Albert-Schweitzer-Str. 33, 48149 Münster, Germany. E-mail: mwild@mpi-muenster.mpg.de or marquat@unimuenster.de. 
platelets results in their clearance from the circulation. ${ }^{5}$ Moreover, mice deficient for $\alpha 2,3$-sialyltransferase IV show strong thrombocytopenia. ${ }^{6,7}$ Interestingly, as in CDG-IIf, platelets are enlarged in these mice. Recently, experiments in mice have shown that hyposialylated platelets readily bind to the liver asialoglycoprotein receptor (ASGP-R) in vivo and are efficiently removed from the circulation by the ASGP-R, explaining the induction of thrombocytopenia. ${ }^{8,9}$ Here, we present the first type of human macrothrombocytopenia that is associated with a specific defect of $\alpha 2,3$-sialylation and with strong platelet binding to the ASGP-R.

\section{Materials and Methods}

\section{Flow Cytometry}

Flow cytometry was performed according to standard protocols. ${ }^{10}$ Anti-sLe ${ }^{\times}$antibody CSLEX-1 (American Type Culture Collection, Manassas, VA), anti-Le $\mathrm{e}^{\mathrm{x}}$ antibody (BD Pharmingen, Heidelberg, Germany), negative control IgM monoclonal antibody (mAb) (BD Pharmingen), antiGQ1b/GD3 mAb R24, ${ }^{11}$ anti-PSA mAb 735, ${ }^{12}$ anti-Le ${ }^{a}$ antibody (Acris Antibodies, Hiddenhausen, Germany), and anti-GP1b mAb AK2 (Serotec, Düsseldorf, Germany) were used at $10 \mu \mathrm{g} / \mathrm{mL}$. Biotinylated Aleuria aurantia lectin (AAL), concanavalin $A$, peanut agglutinin, Sambucus nigra lectin (SNA), and Maackia amurensis lectin II (MAL II) (Vector Laboratories, Burlingame, CA) were used at 2 to $5 \mu \mathrm{g} / \mathrm{mL}$ and detected with phycoerythrin-conjugated streptavidin (Jackson Immunoresearch, West Grove, PA). E- and P-selectin- $\mathrm{Fc}^{13}$ were used as described. ${ }^{10} \mathrm{~A}$ total of $0.2 \mu \mathrm{g}$ murine myelin-associated glycoprotein (MAG)-Fc and human CD22-Fc (prepared as in ${ }^{14}$ ), respectively, were incubated with phycoerythrin-conjugated donkey anti-human IgG (0.2 $\mu \mathrm{g}$; Jackson Immunoresearch) in $50 \mu \mathrm{L}$ of PBS for 1 hour and added to the cells after 10 minutes of Fc receptor blockage. IL-8 binding to granulocytes was tested by incubating the cells with different concentrations of a fluorescently labeled human IL-8 peptide [K69(CF)]hIL-8(1-77) containing amino acids 1-77 and carboxyfluorescein attached to lysine $69^{15}$ in PBS/0.1\% bovine serum albumin for 30 minutes at $4^{\circ} \mathrm{C}$ before cells were washed, fixed, and then analyzed by flow cytometry. For control, cells were treated with $100 \mathrm{mU} / \mathrm{mL}$ neuraminidase (from Vibrio cholerae; Sigma) for 30 minutes at $37^{\circ} \mathrm{C}$ in $\mathrm{PBS} / 0.1 \%$ bovine serum albumin before they were washed three times and were incubated with IL-8.

Platelet analysis was done with the following precaution: For comparison of control and patient platelets, we gated on a very small window in the forward scatter/ sideward scatter where large normal platelets and small patient platelets overlap in size to exclude size effects. To test for ASGP-R binding, platelets were incubated with $2.5 \mu \mathrm{g} / \mathrm{mL}$ ASGP-R (purified from human liver as described ${ }^{16}$ ) for 1 hour at $4^{\circ} \mathrm{C}$, washed and incubated with anti-ASGP-R mAb (30201; Calbiochem, San Diego, CA). To distinguish patient and donor platelets within the patient samples, the $\mathrm{Le}^{\mathrm{a}}$-positive patient received $\mathrm{Le}^{\mathrm{a}}$-neg- ative thrombocytes. Patient and donor platelets were distinguished with an anti-Le ${ }^{a}$ antibody (Acris Antibodies). Values obtained by flow cytometry give mean fluorescence intensity $(\mathrm{MFI}) \pm \mathrm{SD}$ from at least three experiments.

\section{Blood-Perfused Flow Chamber Assays}

Leukocyte rolling was tested with whole human blood in a blood-perfused flow chamber system ${ }^{17}$ in which rectangular glass capillaries were coated with $5 \mu \mathrm{g} / \mathrm{mL}$ human E- and $20 \mu \mathrm{g} / \mathrm{mL}$ human P-selectin-Fc proteins (both from R\&D Systems, Abingdon, UK), respectively, for 2 hours and then blocked for 1 hour using casein. Slow rolling was obtained by co-immobilizing $\mathrm{E}$ - and P-selectin with intercellular cell adhesion molecule-1 (ICAM-1) 3 and 5 $\mu \mathrm{g} / \mathrm{mL}$, respectively). Representative fields of view were recorded for 1 minute using a SW40/0.75 objective and a digital camera (Sensicam QE; Cooke Corporation, Kelheim, Germany). Results were normalized according to the leukocyte numbers of the blood samples.

\section{Isoelectric Focusing of Serum Transferrin and Apolipoprotein C-III}

Transferrin was immunoprecipitated from serum and stained. ${ }^{18}$ Isoelectric focusing was performed with a Phast electrophoresis system using a gel with a $\mathrm{pH}$ range of 5 to 7 (Pharmacia, Freiburg, Germany). Isofocusing of apolipoprotein C-III (ApoCIII) was performed as described $^{19}$ using a mixture of pharmalytes (4.2 to 4.9 and 2.5 to 5; GE Healthcare, Freiburg, Germany), Western blotting with purified anti-ApoCIII Ab (Rockland, Gilbertsville, PA) and relative quantification of the Enhanced chemiluminescence signal on a Fujifilm Luminescent Image Analyzer (Fuji Photo Film, Tokyo, Japan).

\section{Fibroblast Transfection}

Fibroblasts were transfected with mouse $\alpha 1$,3-fucosyltransferase VII DNA in vector pcDNA3 ${ }^{20}$ by nucleofection as described. ${ }^{21}$

\section{Mutation Analysis and Real-Time PCR of Sialyltransferases}

Total RNA (2 $\mu \mathrm{g}$ ) was isolated from blood leukocytes (for mutation analysis) or monocytes (for real-time PCR) using a RNAeasy Mini Kit (Qiagen, Hilden, Germany). cDNA was obtained with SuperScript Reverse Transcriptase III (Invitrogen, Karlsruhe, Germany). Sequencing was done using an ABI Prism 3700 capillary sequencer and BigDye 3.1 (Applied Biosystems, Darmstadt, Germany). Quantitative PCR was performed with appropriate primers in an ABI PRISM 7900HT device (Applied Biosystems) using the QuantiTect SYBR Green PCR Master Mix (Qiagen). Reactions were performed in reference to housekeeping genes cyclophilin $\mathrm{A}, h A L U$, and $G A P D H$ and compared to control cDNA samples from four healthy donors. 


\section{Mutation Analysis of the CMP-Sialic Acid Transporter}

Genomic DNA was prepared from EDTA-treated whole blood samples of the patient and healthy control donors using the QiaAmp Blood Kit (Qiagen). For generation of cDNA, RNA was prepared from whole blood using the PaxGene Blood RNA Kit (Qiagen) or from fibroblasts using the RNAEasy Kit (Qiagen) and was used directly for PCR. All eight exons of the genomic Slc35a1 sequence were amplified with the following forward and reverse primers binding to flanking intron sequences: exon 1, 5'-GCGGGGAGACGCAGTTTACA-3', , 5'-CACTCCCAGCTAGTGGAGGT-3'; exon 2, 5'-GCAATTGGGGCACTCCCTAG-3', 5'-GCTTTGCAAGCCAGTCAGTC-3'; exon 3 plus 4, 5'-CACTTGAACACGGGAGGTG-3', 5'-CCTCTTTGGGGACTGTCATC-3'; exon 5, 5'-GGAGTCTTAAGAGGCAGCAC-3', 5'-GCCGGACTGAGATGGTCAAG-3'; exon 6 plus intron 6 plus exon 7, 5'-GTCATGTGTCACACAACCTAC-3', 5'-CTAGCACCCCTCGTCTTAAC-3'; exon 8, 5'-CTTGATTTTACCCGCCCTGC-3', 5'-GTAGACCCCAAACAGGTCTA-3'. PCR conditions were 5 minutes at $94^{\circ} \mathrm{C}, 35$ cycles with 1 minute at $94^{\circ} \mathrm{C}, 1$ minute at $67^{\circ} \mathrm{C}, 1.5$ minutes at $72^{\circ} \mathrm{C}$, and 5 minutes at $72^{\circ} \mathrm{C}$. PCR of cDNA was performed with primers 5'-GTACAGTGGAAACCAGCCCA-3' (bp 499 in NCBI accession No. D87969) and 5'-GTAGACCCCAAACAGGTCTA-3' (bp 1247) amplifying a fragment of 749 bp starting in exon 4 and ending downstream of the stop codon of Slc35a1 (annealing at $68^{\circ} \mathrm{C}$ for 2 minutes). PCR products were treated with the PCR product presequencing kit (USB Corporation, Cleveland, $\mathrm{OH}$ ) before they were sequenced as described before. ${ }^{22}$

\section{Electron Microscopy}

Isolated platelets were fixed in $2 \%$ paraformaldehyde, $0.2 \%$ glutaraldehyde in $0.1 \mathrm{~mol} / \mathrm{L}$ phosphate buffer and were processed further for ultrathin cryosectioning and immunogold labeling as described. ${ }^{23}$

\section{Consent to Patient Studies}

Studies on the patient's cells, bone marrow biopsies, and publication of results were done with written consent of the patient's parents and approval by the Ethics Commission of the University of Münster.

\section{Results}

\section{A Novel Disorder with Severe Thrombocytopenia and Variable Neutropenia}

We detected the novel disease in a girl who developed spontaneous bleeding within the first day of life. The neonate was diagnosed with thrombocytopenia $(12,000$ to 17,000 platelets/ $\mu \mathrm{L}$ blood; normal range: 140,000 to $300,000 / \mu \mathrm{L}$ ) and strong initial neutropenia (120 neutrophils/ $\mu \mathrm{L}$ blood; normal range: 1250 to $6500 / \mu \mathrm{L}$ ). Whereas the severity of the thrombocytopenia remained stable over more than 8 years since the patient's birth, the neutropenia showed considerable variability, with neutrophil numbers ranging from 70 to $5700 / \mu \mathrm{L}$ and a gradual normalization (see Supplemental Tables S1 and S2 at http://ajp.amjpathol. org). At the age of 8 years, the patient shows neutrophil counts of 1200 to $3300 / \mu \mathrm{L}$ and is thus no longer neutropenic. Higher neutrophil counts were recorded during bacterial infections. Despite variable neutropenia, the child never showed signs of immunodeficiency. Counts of lymphocytes, monocytes, eosinophils, and basophils were always within the normal range (see Supplemental Tables S1 and S2 at http://ajp.amjpathol.org).

Starting with day 1 after birth, the thrombocytopenia had to be controlled by weekly platelet transfusions. No further bleeding events were observed under this substitution regime for the 8 years she has been studied. Von Willebrand disease was excluded and no auto- or allo-antibodies against thrombocytes or granulocytes could be detected in the patient. Further details on the phenotype are given in Table 1.

\section{Giant Platelets with Ultrastructural Abnormalities}

Flow cytometry analysis of peripheral blood cells revealed the presence of normal-sized thrombocytes as well as of giant platelets. This was confirmed by electron microscopy (Figure 1). The enlarged platelets had a mean diameter of $5.0 \mu \mathrm{m}$ with a range of 3.5 to $6.3 \mu \mathrm{m}$ (control platelets mean: $2.5 \mu \mathrm{m}$, range: 1.8 to $2.8 \mu \mathrm{m}$ )

Table 1. Phenotype of the Novel Thrombocytopenic Disorder

Patient: girl, born in 2002 to nonconsanguineous parents, uneventful pregnancy

Normal weight, length, and head circumference

Petechial bleedings at trunk and legs, bloody vomiting, and small intracerebral bleeding $\left(I^{\circ}\right) 12$ hours after birth

Initial thrombocytopenia of 12,000 thrombocytes per $\mu \mathrm{L}$

No antibodies against GP IIb/IIla, la/lla, Ib/IX, and HLA in maternal serum

No antibodies on the surface of patient platelets, giving no indication of neonatal alloimmune thrombocytopenia

Abundant megakaryocytes with normal to decreased size and normal neutrophil precursor cells in bone marrow biopsies

Enlarged thrombocytes of heterogenous size with giant thrombocytes nearly as large as erythrocytes in peripheral blood

Neutrophil counts below 1000 per $\mu \mathrm{L}$ at 4 weeks of age and below 500 per $\mu \mathrm{L}$ on several occasions thereafter

No antibodies against granulocytes detectable

Thrombocytopenia and neutropenia unresponsive to corticosteroid treatment for 2 weeks and to immunoglobulin treatment

No further bleeding complications due to weekly thrombocyte transfusions

Thrombocyte counts constantly below 10,000 per $\mu \mathrm{L}$ upon admission

Despite neutropenia no increased frequency of infections

No splenomegaly

Normal psychomotor development 

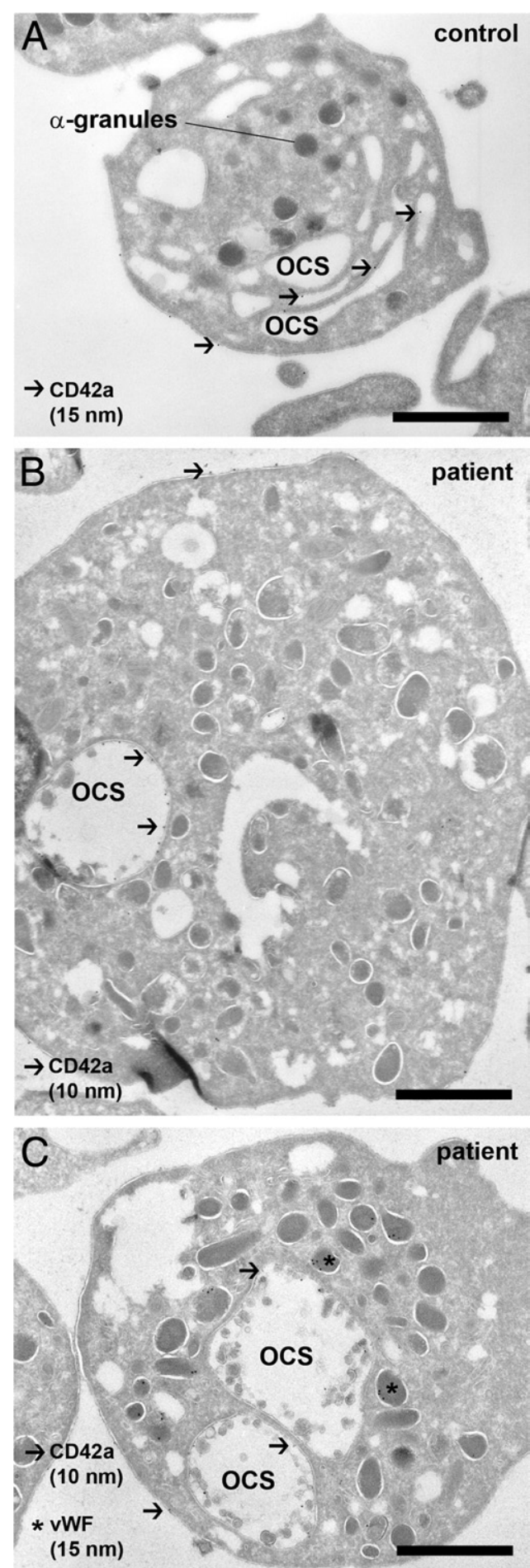

Figure 1. Platelets are enlarged and show changes of the open canalicular membrane system. A: Typical appearance of a normal resting human platelet in an ultrathin cryosection. The cell has a round shape, and $\alpha$-granules are filled with secretory cargo. The open canalicular membrane (OCS) is continuous with the plasma membrane and appears as a cluster of segmented channels, positively identified by an anti-CD 42a $\mathrm{mAb}$ and $15 \mathrm{~nm}$ protein A gold (arrows). B and $\mathbf{C}$ show representative patient platelets at the same magnification. The diameter of the platelets is increased, and the OCS, labeled for CD $42 \mathrm{a}$, is condensed to one or two large saccular compartments. The round shape of the platelets and the presence of von Willebrand factor (vWF) in $\alpha$-granules show that the platelets are quiescent. Bound anti-CD $42 \mathrm{a}$ mAb was detected with $10-\mathrm{nm}$ protein A gold (arrows), and anti-VWF Ab with 15-nm protein A gold (asterisks). Scale bar $=1 \mu \mathrm{m}$. and a calculated mean volume of $65 \mathrm{fL}$ with a range of 30 to $80 \mathrm{fL}$ (control platelets: $8 \mathrm{fL}$, range: 5 to $12 \mathrm{fL}$ ).

Ultrastructural analysis showed striking abnormalities of the platelets' open canalicular membrane system (OCS). Here, the multiple segmented channels that form the OCS in normal platelets (Figure $1 \mathrm{~A}$ ) were replaced by one or two large saccular compartments (Figure 1, B and $\mathrm{C})$, strongly indicating a defect in platelet formation. The micrographs also show sphere-like structures within the OCS of the patient platelets, but not of control platelets. Some of these structures appear to be surrounded by membranes. Abnormal membrane blebbing of the OCS may be an explanation for this phenomenon. Bone marrow examination on three separate occasions showed that erythroid and granulocytic maturation were largely normal with a marginal left shift. Interestingly, megakaryocytic hyperplasia with an increased number of micromegakaryocytes was found (not shown).

\section{Binding of Selectins and IL-8 to Granulocytes}

To analyze the molecular cause for this disease, we turned to granulocytes that were obviously also affected but considerably easier to obtain from the patient than platelets. When we analyzed the expression of adhesion molecules in patient granulocytes, we noticed that binding of soluble E- and P-selectin-Fc fusion proteins was reduced by $70 \% \pm 14 \%$ and $76 \% \pm 15 \%$, respectively [mean fluorescence intensity (MFI) values \pm SD from five independent flow cytometry experiments] (Figure 2A). This defect, however, was not as strong as in leukocyte adhesion deficiency II, where the expression of functional selectin ligands and leukocyte rolling on selectins are virtually absent. ${ }^{10,24}$ Indeed, the number of patient and control leukocytes rolling on immobilized E- and P-selectin in flow chamber assays showed no significant difference (Figure 2B). Moreover, the velocities of cells rolling on selectins or on selectins plus intercellular adhesion molecule-1 (slow rolling) were identical for patient and control leukocytes (Figure 2C). These data suggest that the residual selectin ligands that we detected in patient cells are sufficient to preserve selectin-mediated interactions. Finally, we tested binding of interleukin-8 (IL-8) to granulocytes since the interaction of this leukocyte arrest-mediating chemokine was found to be reduced in mice deficient for $\alpha 2,3$-sialyltransferase IV and in human cells treated with neuraminidase..$^{25}$ Figure $2 \mathrm{D}$ shows that binding of human IL-8 to patient granulocytes over a concentration range of 10 to $90 \mathrm{nmol} / \mathrm{L}$ was only marginally reduced (MFI: $84 \% \pm$ $29 \%$ of control values, $n=9$ ). In fact, only at the highest IL-8 concentration a reduction was detected (an example is shown in the Figure 2D). Taken together, the data on selectin-mediated rolling and IL-8 binding are consistent with the finding that the patient shows normal immunity.

\section{Myeloid Cells and Platelets Display a Defect in $\alpha 2,3-$ Sialylation}

Selectin ligands carry fucosylated and sialylated glycostructures that are identical with or similar to sialyl-Lewis $X$ 
A

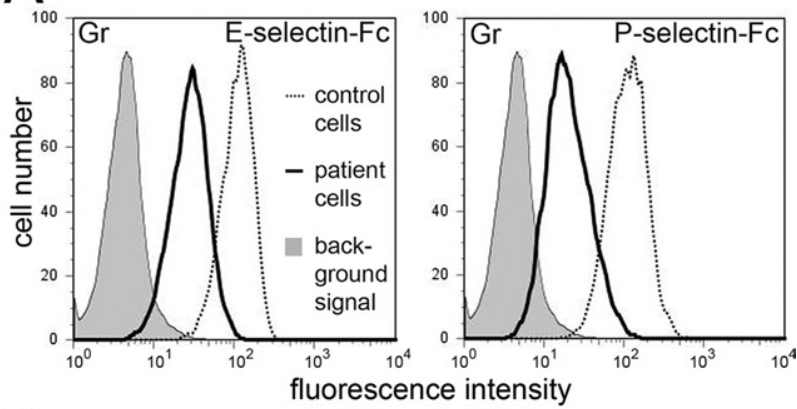

$\mathrm{B}$

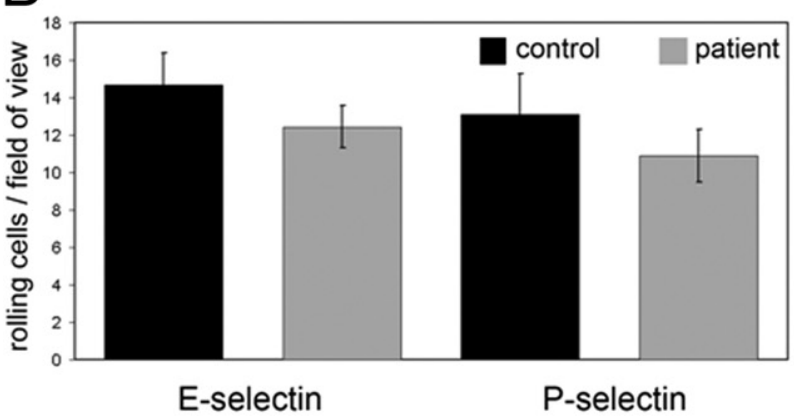

C
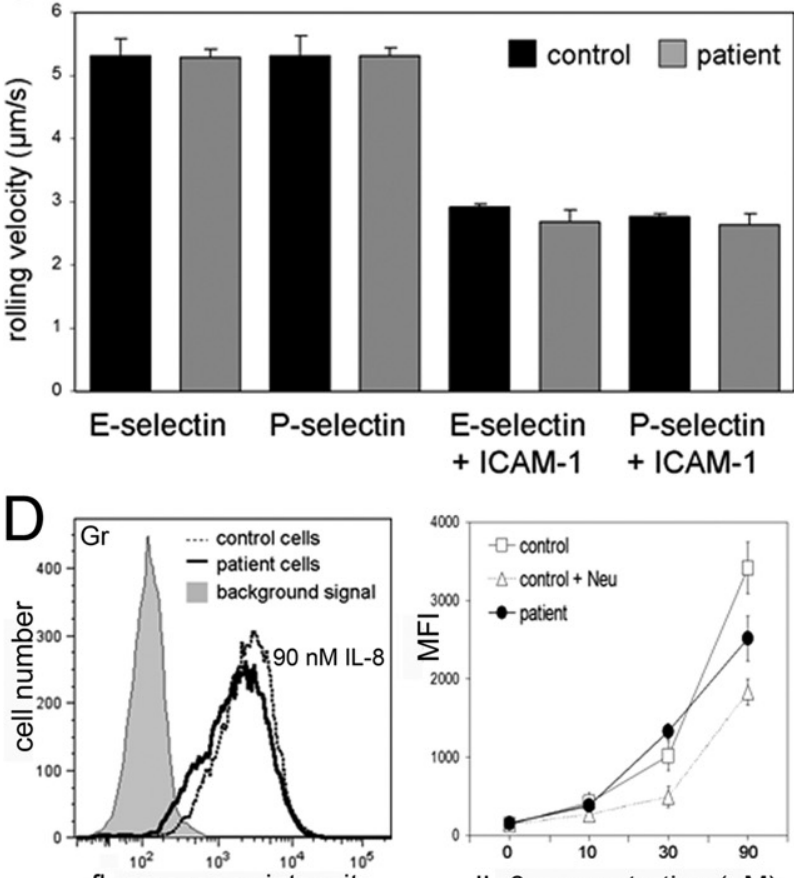

fluorescence intensity

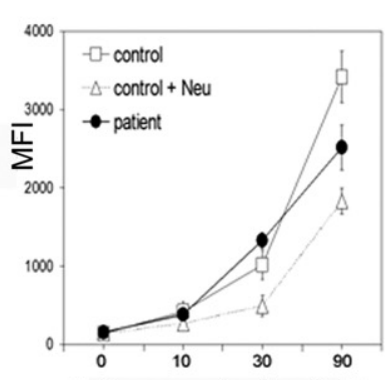

IL-8 concentration ( $\mathrm{nM}$ )

Figure 2. Hyposialylation only marginally affects binding of selectins and IL-8. A: Binding of E- and P-selectin-Fc chimeric proteins to granulocytes. Background signals were obtained with VE-cadherin-Fc and were virtually identical in control and patient cells. The latter are shown. Results are representative for three experiments. B: Leukocyte rolling on endothelial selectins. The number of rolling leukocytes in whole blood on immobilized $\mathrm{E}$ - and P-selectin was assessed in a blood-perfused flow chamber. Nine fields of view were analyzed in each sample. Data are from three independent experiments. C: Rolling velocities. Leukocytes were analyzed as in B. Additionally, slow rolling on selectins co-immobilized with ICAM-1 was analyzed. Data are from three independent experiments. D: Binding of human IL-8 to granulocytes. Cells were incubated with a fluorescently labeled human IL-8 peptide. As control, cells were incubated with neuraminidase from Vibrio cholerae. Neuraminidase treatment reduced IL- 8 binding only partially as has been shown before. ${ }^{25}$ The left panel shows representative histograms. Data in the right panel are from three independent experiments.

$\left(s L e^{x}\right)$ and are required for selectin binding. We found that patient granulocytes exhibited strongly reduced surface expression of $\mathrm{sLe}^{\times}(19 \% \pm 9 \%$ of control MFI, $n=8)$ and increased expression of its nonsialylated form $\operatorname{Le}^{x}(463 \% \pm$ $96 \%$ of control MFI, $n=8$ ) (Figure 3A). Similar results were obtained for blood monocytes (not shown).

We then studied lectin binding to granulocytes and found normal binding of concanavalin A and AAL, which are specific for $\alpha$-linked mannose and fucose, respectively (not shown). However, the cells showed increased binding of peanut agglutinin (PNA) which binds to the nonsialylated, but not to the sialylated, form of the $T$ antigen (galactosyl- $\beta 1,3-\mathrm{N}$-acetylgalactosamine), suggesting a defect in T antigen sialylation (see Supplemental Figure S1 at http://ajp.amjpathol.org). More interestingly, we found that binding of Maackia amurensis lectin II (MAL II), which preferentially recognizes sialic acid linked to galactose in $\alpha 2,3$-linkage, was reduced (Figure 3B). In contrast, binding of SNA, which preferentially recognizes $\alpha 2$,6linked sialic acid, was normal (Figure 3B). Together with the reduced expression of the $\alpha 2,3$-sialylated sLe $e^{\times}$, these data strongly suggested a partial $\alpha 2,3$-sialylation defect.

Hypo- $\alpha 2,3$-sialylation in granulocytes was confirmed by reduced binding of recombinant myelin-associated glycoprotein (MAG/Siglec-4), which is specific for $\alpha 2,3-$ linked sialic acid, whereas binding of $\alpha 2,6$-sialylationspecific CD22 (Siglec-2) was normal (Figure 3C). This pattern of defective $\alpha 2,3$-sialylation and normal $\alpha 2,6$-siaIylation was also found in peripheral blood monocytes (not shown). In control lymphocytes, $\mathrm{Le}^{\mathrm{x}}$ and $\mathrm{SLe}^{\mathrm{x}}$ were hardly detectable, but MAL II and MAG readily bound and gave equal signals in control and patient cells, showing that the defect in $\alpha 2,3$-sialylation is not present in these cells (data not shown). As skin fibroblasts are devoid of sLe ${ }^{x}$, we transfected them with a plasmid containing the cDNA sequence coding for $\alpha 1,3$-fucosyltransferase VII. This led to virtually equal expression of $s \mathrm{~L}^{\mathrm{x}}$ in healthy and patient fibroblasts (not shown), demonstrating that skin fibroblasts are not affected by the disease and that the defect is cell-specific.

In contrast to $\alpha 2,3$-sialylation, the third type of sialic acid linkage, $\alpha 2,8$-sialylation, was found to be normal in granulocytes as judged by normal binding of mAb CGM3, which reacts with the $\alpha 2,8$-sialylated gangliosides GQ1b and GD3 (Figure 3D). Binding of mAb 735, which is specific for $\alpha 2,8$ sialylated polysialic acid, bound very weakly to control granulocytes (not shown). However, it showed detectable and equal binding to control and patient monocytes (Figure $3 \mathrm{D}$ ), further excluding a defect in $\alpha 2,8$-sialylation.

Flow cytometry analysis of control and patient thrombocytes showed that E- and P-selectin as well as antibodies against $s L e^{x}, L e^{x}, G Q 1 b / G D 3$, and polysialic acid did not bind to these cells. However, we found that, as in granulocytes, binding of $\alpha 2,3$-sialylationspecific MAL II and MAG, but not of $\alpha 2,6$-sialylationspecific SNA and CD22, to patient platelets was reduced (Figure 4, A and B). Binding of fucose-specific AAL was normal (Figure 4C).

Changes in the sialylation patterns of serum transferrin and/or apolipoprotein C-III had been shown for all types of CDG-I as well as CDG-lla, -d, -e, -f. ${ }^{26}$ 

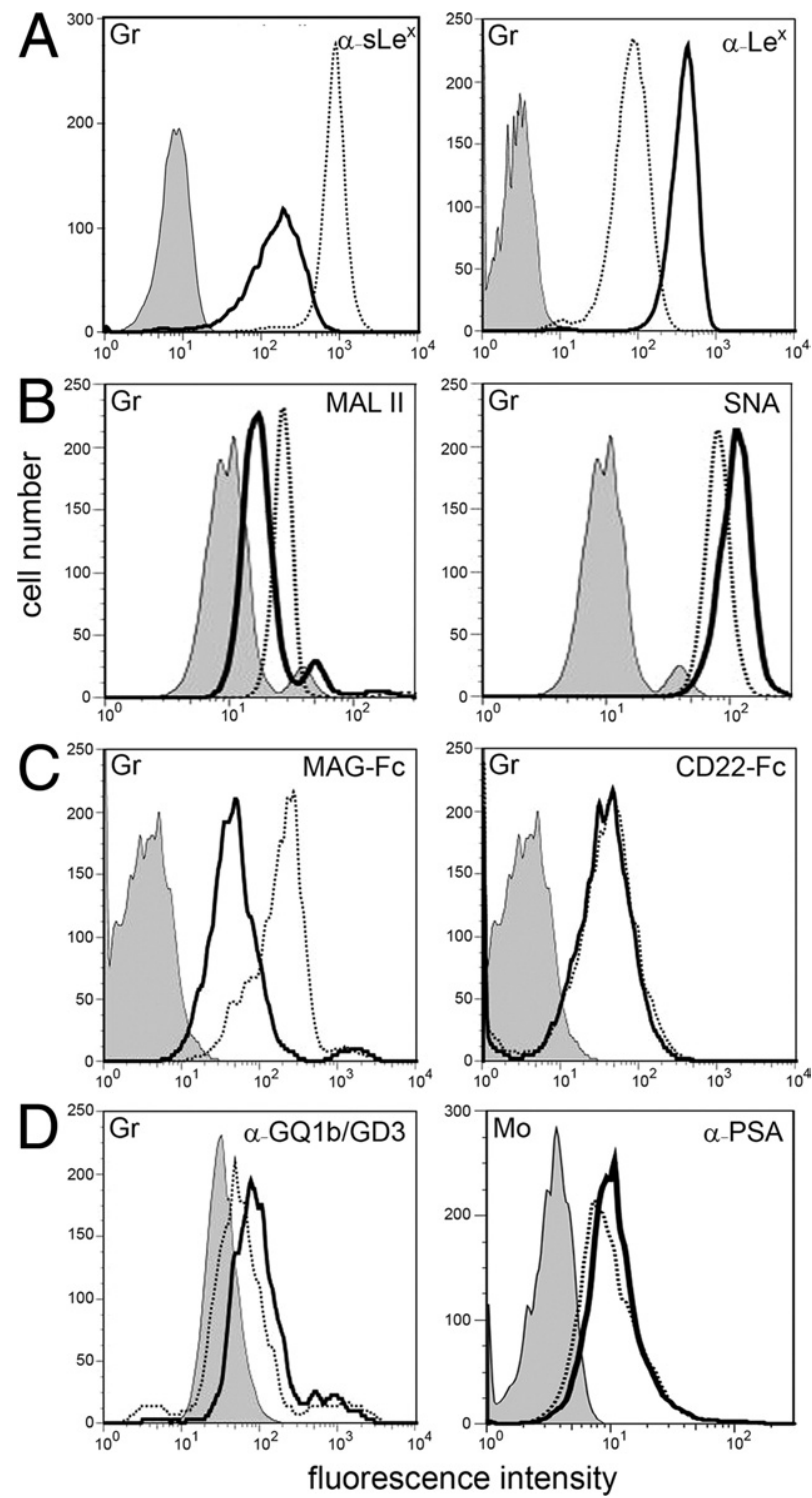

Figure 3. Myeloid cells display a defect in $\alpha 2,3$-sialylation. Granulocytes (Gr) were tested for binding of the following reagents: mAbs against sLe ${ }^{\mathrm{x}}$ and $\mathrm{Le}^{\mathrm{x}}$ (A), Maackia amurensis lectin II (MAL II, binds preferentially to $\alpha 2,3$ sialylated structures) and Sambucus nigra lectin (SNA, binds preferentially to $\alpha 2,6$-sialylated structures) (B), myelin-associated glycoprotein-Fc chimera (MAG-Fc, Siglec-4-Fc; binds to $\alpha 2,3$-sialylated structures) and CD22-Fc (Siglec-2-Fc; binds to $\alpha 2,6$-sialylated structures) (C), and a mAb that detects $\alpha 2,8$-sialylated gangliosides GQ1b and GD3 (D, left panel). Binding of a mAb specific for $\alpha 2,8$-sialylated polysialic acid (PSA) to monocytes (Mo) is show in D (right panel). Background signals were obtained with isotype control mAbs (for mAbs), secondary reagent only (for lectins), and VEcadherin-Fc (for Fc-constructs). Background signals in control and patient cells were virtually identical. The latter are shown. Results are representative for at least three experiments per panel.

However, isoelectric focusing of the patient's serum transferrin and apolipoprotein C-III revealed normal sialylation (Figure 5 and Table 2). In addition, defects in the generation of the lipid-linked oligosaccharide (LLO), which are indicative of CDG-I, were excluded by high-performance liquid chromatography analysis (see Supplemental Figure S2 at http://ajp.amjpathol.org). Taken together, these data show that the novel disease is characterized, not by a general sialylation defi- ciency, but rather by a specific defect in $\alpha 2,3$-sialylation in a restricted set of cells.

\section{No Functional Mutations in the Gene Coding for the CMP-Sialic Acid Transporter}

We performed a large number of experiments in search for the genetic defect causing this disease. We reasoned that with impaired $\alpha 2,3$-sialylation and reduced $s L e^{x}$ expression, an $\alpha 2,3$-sialyltransferase (ST3-Gal) may be defective. Three $\alpha 2,3$-sialyltransferases (ST3-Gal III, IV, VI) can potentially generate $s L e^{\times} .27,28$ We sequenced exon sequences with flanking intron regions of the enzymes' genomic DNA obtained from patient monocytes. No mutations were detected (not shown). We also performed real-time PCR and found that mRNA expression levels of the three sialyltransferases in patient monocytes were completely normal (not shown). As the disease described here shows similarities with CDG-IIf, we also analyzed the gene coding for the CMP-sialic acid
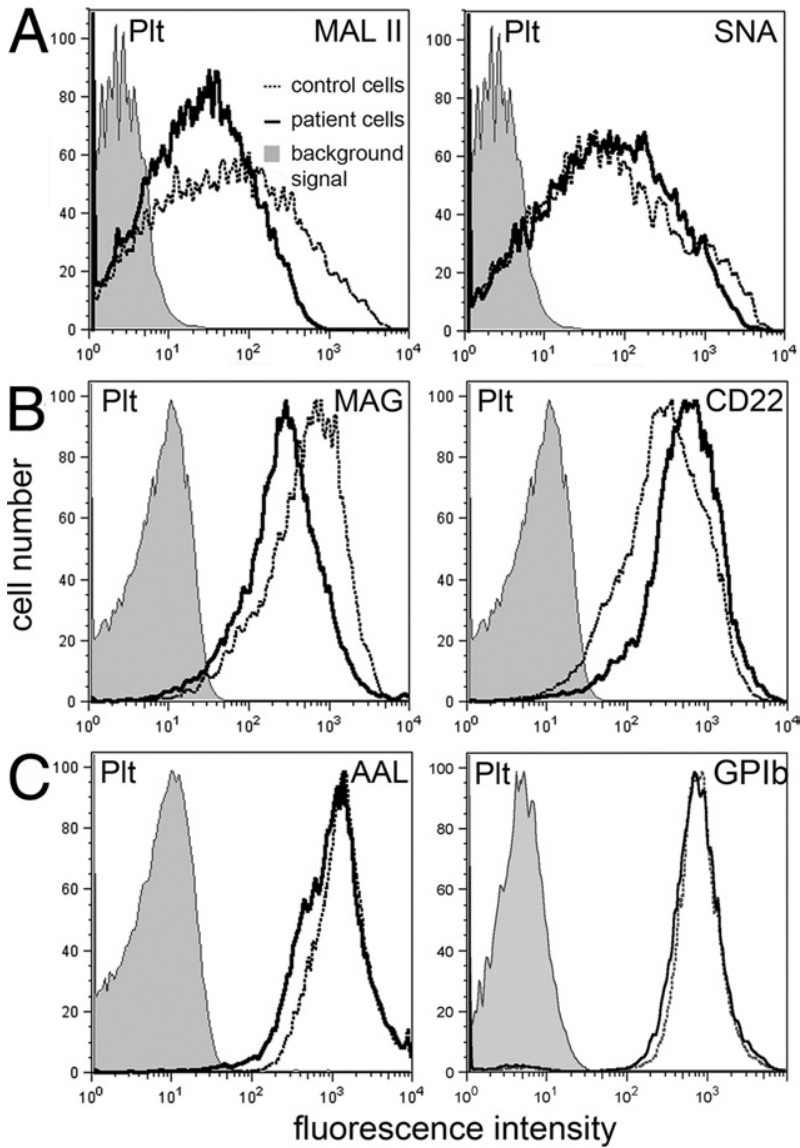

Figure 4. Defect in $\alpha 2,3$-sialylation but normal GPIb expression in platelets Platelets (Plt) were tested for binding of the following reagents: Maackid amurensis lectin II (MAL II) and Sambucus nigra lectin (SNA) (A), myelinassociated glycoprotein-Fc (MAG-Fc, Siglec-4-Fc) and CD22-Fc (Siglec-2-Fc) chimeras (B), Aleuria aurantia lectin (AAL) (C; left panel), and a GPIb mAb (C; right panel). Background signals were obtained with isotype control mAbs (for mAbs), secondary reagent only (for lectins), and VE-cadherin-Fc (for Fc-constructs). Histograms were obtained from a very small window in the forward scatter/sideward scatter plot where large normal platelets and small patient platelets overlap in size to exclude size effects. Results are representative for at least three experiments per panel. 


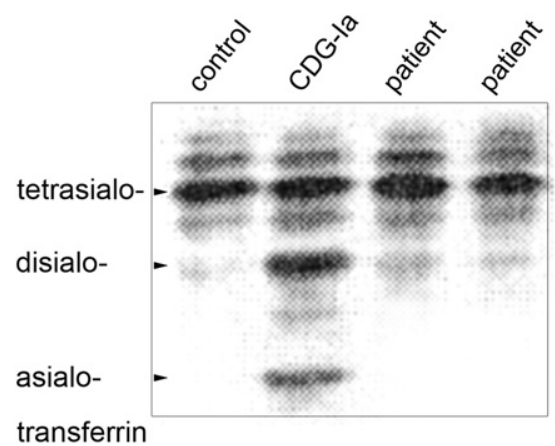

Figure 5. Normal sialylation of serum transferrin. Isoelectric focusing of serum transferrin. Two independently collected serum samples of the patient described in this report, a sample of a CDG-Ia patient and of an age- and sex-matched healthy control person were subjected to IEF. Unlike the CDG-Ia sample, the patient samples show normal distribution of tetra- and disialylated forms of transferrin. One of three experiments is shown.

transporter (SLC35A1). We sequenced all eight exons of the Slc35a1 gene in genomic DNA obtained from peripheral leukocytes of the patient but found no mutations. Sequencing of intron 6 revealed that the patient was heterozygous for an insertion of four nucleotides (CACT) between bp 40958 and 40959 (NCBI accession number NG_016207.1) (Figure 6A and see Supplemental Figure S3 at http://ajp.amjpathol.org). The same insertion had been found in the mother of the CDG-IIf patient. It was predicted to cause one of the two mutations found in the CDG-IIf patient, a 130-bp deletion in exon 6, by modifying the splicing of this exon. ${ }^{4}$ However, analysis of cDNA of our patient by PCR that covered exons 5 to 8 led to PCR products with the expected normal length of $749 \mathrm{bp}$ (Figure 6B), and cDNA sequencing confirmed that no deletions of the Slc35a1 coding sequence were present (see Supplemental Figure S4 at http://ajp.amjpathol.org). Finally, we searched for the CACT insertion in genomic DNA samples of 51 healthy individuals and found that 19 samples were heterozygous and 6 were homozygous for this mutation. This demonstrates that the insertion in intron 6 of the S/c35a1 gene represents a silent polymorphism. Taken together, these data exclude the presence of a genetic defect of the CMP-sialic acid transporter,

Table 2. Isoelectric Focusing of Apolipoprotein CIII

\begin{tabular}{cccc}
\hline $\begin{array}{c}\text { Percentage of } \\
\text { ApoCIII isoforms* }\end{array}$ & $\begin{array}{c}\text { ApoCIII-0 } \\
(\%)\end{array}$ & $\begin{array}{c}\text { ApoCIII-1 } \\
(\%)\end{array}$ & $\begin{array}{c}\text { ApoCIII-2 } \\
(\%)\end{array}$ \\
\hline Patient, sample 1 & 5.3 & 68.4 & 26.4 \\
Patient, sample 2 & 6.5 & 60.4 & 33.2 \\
Patient, sample 3 & 9.7 & 56.5 & 33.7 \\
CDG-IIf patient & 6.0 & 78.0 & 16.0 \\
Reference range & $0-11.6$ & $33.1-66.9$ & $27.4-60.0$ \\
(age: 1-18 yr) & & & \\
Patient's mother & 8.1 & 59.5 & 32.4 \\
Reference range & $2.6-18.9$ & $42.9-69.2$ & $23.2-50.0$ \\
(age: $>$ 18 yr) & & & \\
\hline
\end{tabular}

*Three independently collected serum samples of the patient and samples of the patient's mother were subjected to isoelectrical focusing Results obtained for a CDG-IIf patient ${ }^{26}$ are shown for comparison. The samples of the patient and her mother show isoform distributions that are within the respective reference ranges, whereas the CDG-IIf results indicate hyposialylation of ApoCIII as seen from the shift of disialylated ApoCIII-2 to monosialylated ApoCIII-1.
A
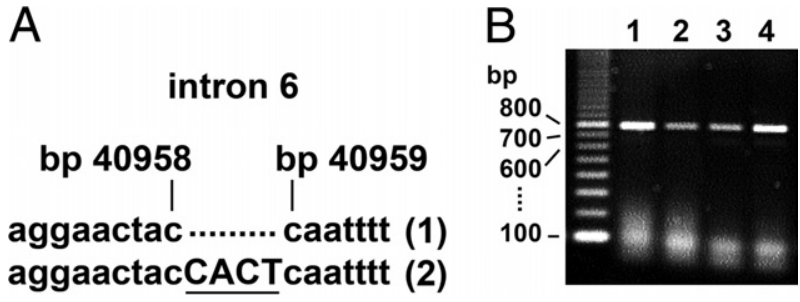

Figure 6. Analysis of the gene coding for the CMP-sialic acid transporte SLC35A1. A: Genomic DNA was prepared from peripheral blood leukocytes of the patient and amplified by PCR. Sequencing results of a fragment containing intron 6 are shown. One allele (1) was found to be normal, the other (2) to contain a CACT insertion between bp 40958 and 40959 (NCBI accession number NG_016207.1). B: Patient (lanes 1, 3) and control (lanes 2, 4) cDNA from blood leukocytes (lanes 1, 2) and fibroblasts (lanes 3, 4) were amplified by PCR covering exons 5 to 8 . All PCR products show the expected normal length of $749 \mathrm{bp}$

leaving the genetic cause of this disease unknown. Platelet glycoprotein GPIb is reduced in CDG-IIf and is reduced or absent in Bernard-Soulier syndrome. ${ }^{3,29,30}$ In contrast to this, the patient's platelets showed normal GPIb expression (Figure 4C), further excluding the presence of one of the above-mentioned diseases.

\section{Abnormal Interaction of Platelets with the Liver Asialoglycoprotein Receptor}

Recently, the liver ASGP-R was shown to remove hypo$\alpha 2,3$-sialylated thrombocytes from the circulation in mice. .,9 $^{-1}$ We therefore analyzed binding of ASGP-R protein purified from human liver to control and patient platelets. Figure 7A shows that the ASGP-R did not bind to control platelets. Thrombocytes of the Le $e^{a}$-positive patient who received platelet transfusions of $\mathrm{Le}^{\mathrm{a}}$-negative donors are depicted in Figure 7B. The figure shows that whereas the ASGP-R protein did not interact with donor platelets, it reacted with virtually all patient platelets. This result suggests that increased platelet binding to the ASGP-R may contribute to the thrombocytopenia in this novel disease.

\section{Discussion}

Our results show that the disease described here is different from known disorders with macrothrombocytopenia. The new sialylation disorder is most similar to CDG-IIf but differs from it in that it shows no alterations of GPIb expression and apolipoprotein C-III glycosylation and lacks mutations of the gene coding for the CMP-sialic acid transporter. The small CATC insert in intron 6 that had been found in the mother of the CDG-IIf patient ${ }^{4}$ was also found in the new patient but turned out to be a silent polymorphism.

Interestingly, the glycosylation defect described here is restricted to $\alpha 2,3$-sialylation, resulting in reduced expression of $\mathrm{SLe}^{\times}$, as well as of ligands for selectins, for MAG and MAL II. This is reminiscent of the phenotype of mice deficient in ST3-Gal IV. ${ }^{6,7}$ These mice show reduced selectin binding to granulocytes and a macrothrombocytopenia that can be counteracted by ASGP-Rblocking asialofetuin, but no neutropenia. Additionally, these mice show reduced serum levels of von Willebrand 

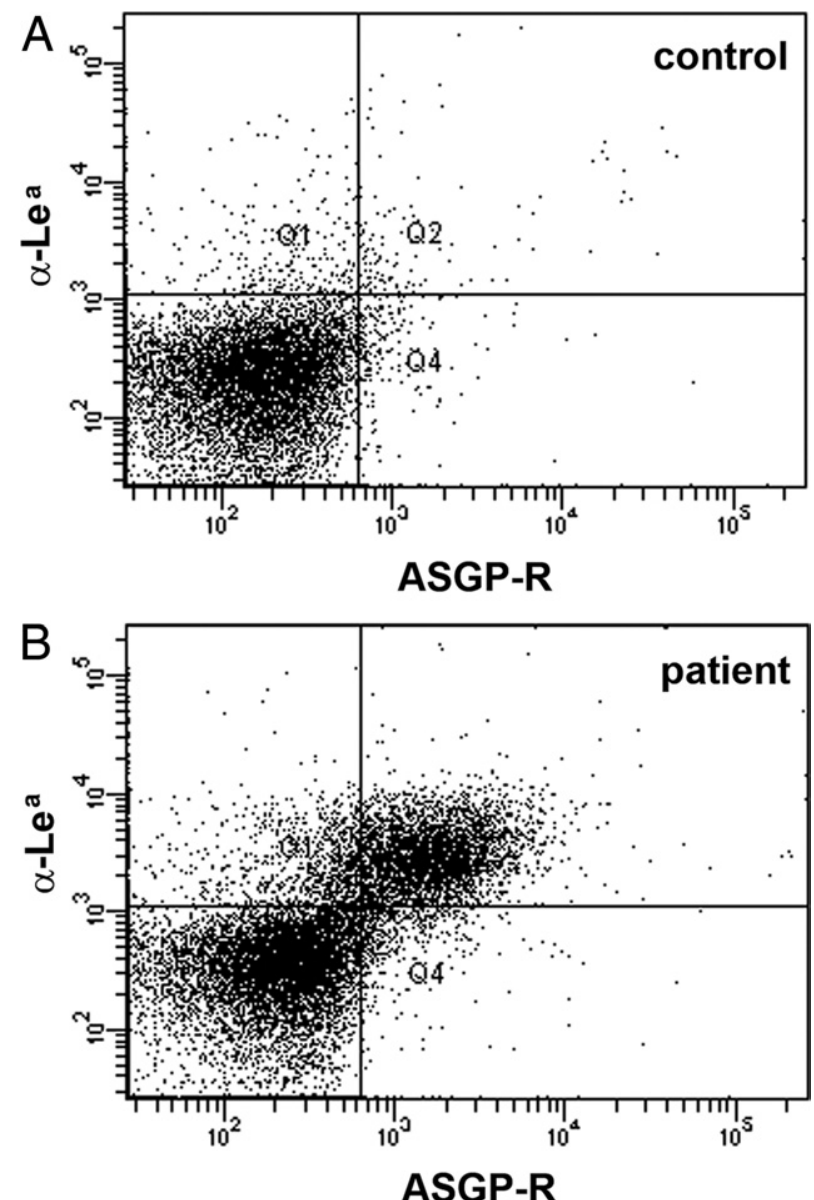

Figure 7. Anomalous platelet interaction with the liver asialoglycoprotein receptor. Platelets were incubated with native purified asialoglycoprotein receptor (ASGP-R) protein before cells were incubated with anti-ASGP-R $\mathrm{mAb}$ and counterstained with anti-Lewis ${ }^{\mathrm{a}}\left(\mathrm{Le}^{\mathrm{a}}\right) \mathrm{mAb}$. A: Platelets of a Le $\mathrm{L}^{\mathrm{a}}$ negative control person are shown. B: Platelets of the Le ${ }^{\mathrm{a}}$-positive patient who received platelet transfusions of Le ${ }^{\mathrm{a}}$-negative donors are shown. Only the patient's platelets, but not the donor platelets, bind to ASPG-R protein. Results are representative for at least three experiments per panel.

factor, which we did not observe in the human disease described here. Despite the similarities between the novel disease and the phenotype of ST3-Gal $N^{-1-}$ mice, we found no mutations and normal gene expression of ST3-Gal IV and two further sialyltransferases that are implicated in the generation of $S L e^{x}$. Thus, the exact molecular cause for this disease remains speculative. One possibility is that ST3-Gal expression is blocked at the translational level. To analyze ST3-Gal protein levels, we tested a ST3-Gal IV-specific antibody (C-19; Santa Cruz Biotechnology, Heidelberg, Germany) which, however, did not give specific signals in a variety of assays including Western blotting and immunofluorescence. To our knowledge, no further antibodies against human ST3Gal proteins are available. Another possibility is that an unknown cofactor that may be required for sialyltransferase activity is defective. However, we were unable to devise an assay that can detect changes in linkagespecific sialyltransferase activity in the low number of affected cells that are available from the patient.
The sialylation defect in this disease is cell-specific. As in ST3-Gal $N^{-1-}$ mice, granulocytes and platelets are affected. In addition, we show that monocytes are defective, whereas lymphocytes and skin fibroblasts are not. This might reflect the expression profile of an ST3-Gal or a cofactor if such proteins are affected in the way described above.

The defect in $\alpha 2,3$-sialylation appears to have an effect on the generation of platelets. We detected an increased number of micromegakaryocytes, indicating defective megakaryocytopoiesis. In addition, patient platelets were enlarged and showed abnormalities of the OCS structure. Furthermore, sphere-like structures were visible within the OCS that may be the result of abnormal membrane blebbing. It is unclear whether this is directly caused by hyposialylation, although alterations in the demarcation process in megakaryocytes have been observed in the sialylation deficiency CDG-IIf, ${ }^{3}$ suggesting that hyposialylation can indeed interfere with membrane formation processes.

How sialylation can influence platelet generation in the bone marrow is not known. Repulsion by the negative charge of sialic acid may be required for a correct demarcation process. On the other hand, interactions of megakaryocytes with E-selectin in the bone marrow appear to be important for megakaryocyte migration, ${ }^{31}$ and P-selectindeficient mice show enhanced megakaryocytopoiesis. ${ }^{32}$ Thus, reduced selectin interactions with hyposialylated ligands might contribute to defective platelet generation.

Desialylation of platelets results in their clearance from the circulation. ${ }^{5}$ Recently, removal of desialylated platelets by liver ASGP-R was found to be a major cause for this phenomenon. ${ }^{8,9}$ Consistent with this, we found that patient platelets showed increased binding to the liver ASGP-R. It is tempting to speculate, but also difficult to prove in patients, that such receptors are at least in part responsible for the thrombocytopenia in the new disease.

We have previously found that the defect in fucosylation in CDG-Ilc can be corrected with oral L-fucose supplementation. ${ }^{10,33} \mathrm{~N}$-acetyl-mannosamine (ManNAc) was described as a sialic acid precursor that can be taken up, metabolized to sialic acid and incorporated into the plasma membrane of mammalian cells. ${ }^{34,35}$ We therefore extensively investigated the effect of exogenous ManNAc (in concentrations ranging from 2 to $50 \mathrm{mmol} / \mathrm{L}$ ) on sLe $\mathrm{e}^{\mathrm{x}}$ expression in cultures of patient granulocytes and monocytes using incubation times of 12 to 72 hours. In neither case did the sialic acid precursor increase $s L e^{x}$ expression (not shown). Thus, thrombocyte transfusion remains the most effective therapy for this novel human sialylation defect.

\section{Acknowledgments}

We thank Ingrid Du Chesne, Marie-Luise Többens (Universitätsklinikum Münster), Martin Stehling and Ute Ipe (the Max Planck Institute for Molecular Biomedicine, Münster) for excellent technical assistance; Paul Crocker (University of Dundee, United Kingdom) for recombinant MAG and CD22 proteins; Rita Gerardy-Schahn (Medical High School, Hannover, Germany) for antibodies; and Heiko Gäthje and Nadine Bock (University of Bremen, Germany) and Karoline Nordsieck (University of Leipzig, 
Germany) for producing Fc constructs and recombinant IL-8, respectively. We thank the Consortium for Functional Glycomics for their support in Glycan analysis, and Carlos Hirschberg (Boston University Goldman School of Dental Medicine, MA) for measuring CMP-sialic acid transport activity in fibroblasts. Above all, we thank the patient for her patience with us.

\section{References}

1. Kunishima S, Kamiya T, Saito H: Genetic abnormalities of BernardSoulier syndrome. Int J Hematol 2002, 76:319-327

2. Seri M, Cusano R, Gangarossa S, Caridi G, Bordo D, Lo Nigro C, Ghiggeri GM, Ravazzolo R, Savino M, Del Vecchio M, D'Apolito M, Iolascon A, Zelante LL, Savoia A, Balduini CL, Noris P, Magrini U, Belletti S, Heath KE, Babcock M, Glucksman MJ, Aliprandis E, Bizzaro N, Desnick RJ, Martignetti JA: Mutations in MYH9 result in the May-Hegglin anomaly, and Fechtner and Sebastian syndromes. The MayHeggllin/Fechtner Syndrome Consortium, Nat Genet 2000, 26:103-105

3. Willig TB, Breton-Gorius J, Elbim C, Mignotte V, Kaplan C, Mollicone R, Pasquier C, Filipe A, Mielot F, Cartron JP, Gougerot-Pocidalo MA, Debili N, Guichard J, Dommergues JP, Mohandas N, Tchernia G: Macrothrombocytopenia with abnormal demarcation membranes in megakaryocytes and neutropenia with a complete lack of sialyl-Lewis-X antigen in leukocytes-a new syndrome? Blood 2001, 97:826-828

4. Martinez-Duncker I, Dupre T, Piller V, Piller F, Candelier JJ, Trichet C, Tchernia G, Oriol R, Mollicone R: Genetic complementation reveals a novel human congenital disorder of glycosylation of type II, due to inactivation of the Golgi CMP-sialic acid transporter. Blood 2005, 105:2671-2676

5. Greenberg J, Packham MA, Cazenave JP, Reimers HJ, Mustard JF: Effects on platelet function of removal of platelet sialic acid by neuraminidase. Lab Invest 1975, 32:476-484

6. Ellies LG, Ditto D, Levy GG, Wahrenbrock M, Ginsburg D, Varki A, Le DT, Marth JD: Sialyltransferase ST3Gal-IV operates as a dominant modifier of hemostasis by concealing asialoglycoprotein receptor ligands. Proc Natl Acad Sci U S A 2002, 99:10042-10047

7. Ellies LG, Sperandio M, Underhill GH, Yousif J, Smith M, Priatel JJ, Kansas GS, Ley K, Marth JD: Sialyltransferase specificity in selectin ligand formation. Blood 2002, 100:3618-3625

8. Grewal PK, Uchiyama S, Ditto D, Varki N, Le DT, Nizet V, Marth JD: The Ashwell receptor mitigates the lethal coagulopathy of sepsis. Nat Med 2008, 14:648-655

9. Sorensen AL, Rumjantseva V, Nayeb-Hashemi S, Clausen H, Hartwig $\mathrm{JH}$, Wandall HH, Hoffmeister KM: Role of sialic acid for platelet life span: exposure of beta-galactose results in the rapid clearance of platelets from the circulation by asialoglycoprotein receptor-expressing liver macrophages and hepatocytes. Blood 2009, 114:1645-1654

10. Marquardt T, Lühn K, Srikrishna G, Freeze HH, Harms E, Vestweber D: Correction of leukocyte adhesion deficiency type II with oral fucose. Blood 1999, 94:3976-3985

11. Pukel CS, Lloyd KO, Travassos LR, Dippold WG, Oettgen HF, Old LJ: GD3, a prominent ganglioside of human melanoma. Detection and characterisation by mouse monoclonal antibody. J Exp Med 1982, 155:1133-1147

12. Frosch M, Görgen I, Boulnois GJ, Timmis KN, Bitter-Suermann D: NZB mouse system for production of monoclonal antibodies to weak bacterial antigens: isolation of an IgG antibody to the polysaccharide capsules of Escherichia coli K1 and group B meningococci, Proc Natl Acad Sci U S A 1985, 82:1194-1198

13. Hahne M, Jager U, Isenmann S, Hallmann R, Vestweber D: Five tumor necrosis factor-inducible cell adhesion mechanisms on the surface of mouse endothelioma cells mediate the binding of leukocytes. J Cell Biol 1993, 121:655-664

14. Kelm S, Pelz A, Schauer R, Filbin MT, Tang S, de Bellard ME, Schnaar RL, Mahoney JA, Hartnell A, Bradfield P, Crocker PR: Sialoadhesin, myelin-associated glycoprotein and CD22 define a new family of sialic acid-dependent adhesion molecules of the immunoglobulin superfamily. Curr Biol 1994, 4:965-972

15. David R, Machova Z, Beck-Sickinger AG: Semisynthesis and application of carboxyfluorescein-labelled biologically active human interleukin-8. Biol Chem 2003, 384:1619-1630
16. Treichel U, Schreiter T, Meyer zum Buschenfelde KH, Stockert RJ: High-yield purification and characterization of human asialoglycoprotein receptor. Protein Expr Purif 1995, 6:251-255

17. Kuwano Y, Spelten O, Zhang H, Ley K, Zarbock A: Rolling on E- or $P$-selectin induces the extended but not high-affinity conformation of LFA-1 in neutrophils. Blood 2010, 116:617-624

18. Niehues R, Hasilik M, Alton G, Korner C, Schiebe-Sukumar M, Koch HG, Zimmer KP, Wu R, Harms E, Reiter K, von Figura K, Freeze HH, Harms HK, Marquardt T: Carbohydrate-deficient glycoprotein syndrome type $\mathrm{lb}$. Phosphomannose isomerase deficiency and mannose therapy. J Clin Invest 101:1414-1420, 1998

19. Wopereis S, Grunewald S, Morava E, Penzien JM, Briones P, GarciaSilva MT, Demacker PN, Huijben KM, Wevers RA: Apolipoprotein C-III isofocusing in the diagnosis of genetic defects in O-glycan biosynthesis. Clin Chem 2003, 49:1839-1845

20. Huang MC, Laskowska A, Vestweber D, Wild MK: The alpha (1,3)-fucosyltransferase Fuc-TIV, but not Fuc-TVII, generates sialyl Lewis X-like epitopes preferentially on glycolipids. J Biol Chem 2002, 277:47786-47795

21. Helmus Y, Denecke J, Yakubenia S, Robinson P, Luhn K, Watson DL, McGrogan PJ, Vestweber D, Marquardt T, Wild MK: Leukocyte adhesion deficiency II patients with a dual defect of the GDP-fucose transporter. Blood 2006, 107:3959-3966

22. Kranz C, Jungeblut C, Denecke J, Erlekotte A, Sohlbach C, Debus V, Kehl HG, Harms E, Reith A, Reichel S, Grobe H, Hammersen G, Schwarzer U, Marquardt T: A defect in dolichol phosphate biosynthesis causes a new inherited disorder with death in early infancy. Am J Hum Genet 2007, 80:433-440

23. Slot JW, Geuze HJ: Cryosectioning and immunolabeling. Nat Protoc 2007, 2:2480-2491

24. Etzioni A, Frydman M, Pollack S, Avidor I, Phillips ML, Paulson JC, GershoniBaruch R: Brief report: recurrent severe infections caused by a novel leukocyte adhesion deficiency. N Engl J Med 1992, 327:1789-1792

25. Frommhold D, Ludwig A, Bixel MG, Zarbock A, Babushkina I, Weissinger M, Cauwenberghs S, Ellies LG, Marth JD, Beck-Sickinger AG, Sixt M, Lange-Sperandio B, Zernecke A, Brandt E, Weber C, Vestweber D, Ley K, Sperandio M: Sialyltransferase ST3Gal-IV controls CXCR2-mediated firm leukocyte arrest during inflammation. $J$ Exp Med 2008, 205:1435-1446

26. Wopereis S, Grunewald S, Huijben KM, Morava E, Mollicone R, van Engelen BG, Lefeber DJ, Wevers RA: Transferrin and apolipoprotein $\mathrm{C}$ - III isofocusing are complementary in the diagnosis of $\mathrm{N}$ - and Oglycan biosynthesis defects. Clin Chem 2007, 53:180-187

27. Okajima T, Fukumoto S, Miyazaki $\mathrm{H}$, Ishida H, Kiso M, Furukawa $\mathrm{K}$, Urano T: Molecular cloning of a novel alpha2,3-sialyltransferase (ST3Gal VI) that sialylates type II lactosamine structures on glycoproteins and glycolipids. J Biol Chem 1999, 274:11479-11486

28. Sasaki K, Watanabe E, Kawashima K, Sekine S, Dohi T, Oshima M, Hanai N, Nishi T, Hasegawa M: Expression cloning of a novel Gal beta (1-3/1-4) GlcNAc alpha 2,3-sialyltransferase using lectin resistance selection. J Biol Chem 1993, 268:22782-22787

29. Jenkins CS, Phillips DR, Clemetson KJ, Meyer D, Larrieu MJ, Luscher EF: Platelet membrane glycoproteins implicated in ristocetin-induced aggregation. Studies of the proteins on platelets from patients with Bernard-Soulier syndrome and von Willebrand's disease. J Clin Invest 57:112-124, 1976

30. Nurden AT, Caen JP: Specific roles for platelet surface glycoproteins in platelet function. Nature 1975, 255:720-722

31. Hamada T, Mohle R, Hesselgesser J, Hoxie J, Nachman RL, Moore MA, Rafii S: Transendothelial migration of megakaryocytes in response to stromal cell-derived factor 1 (SDF-1) enhances platelet formation. J Exp Med 1998, 188:539-548

32. Banu N, Avraham S, Avraham HK: P-selectin, and not E-selectin, negatively regulates murine megakaryocytopoiesis. J Immunol 2002, 169:4579-4585

33. Lühn K, Marquardt T, Harms E, Vestweber D: Discontinuation of fucose therapy in LADII causes rapid loss of selectin ligands and rise of leukocyte counts. Blood 2001, 97:330-332

34. Gu X, Wang DI: Improvement of interferon-gamma sialylation in Chinese hamster ovary cell culture by feeding of $\mathrm{N}$-acetylmannosamine. Biotechnol Bioeng 1998, 58:642-648

35. Kayser H, Zeitler R, Kannicht C, Grunow D, Nuck R, Reutter W: Biosynthesis of a nonphysiological sialic acid in different rat organs, using N-propanoyl-D-hexosamines as precursors. J Biol Chem 1992, 267:16934-16938 